Proyecciones

Vol. 23, No 3, pp. 187-203, December 2004.

Universidad Católica del Norte

Antofagasta - Chile

\title{
ABELIAN AUTOMORPHISMS GROUPS OF SCHOTTKY TYPE
}

\author{
RUBÉN HIDALGO * \\ Universidad Técnica Federico Santa María, Chile \\ Received: April 2004. Accepted : October 2004
}

\begin{abstract}
We study the problem of lifting an Abelian group $H$ of automorphisms of a closed Riemann surface $S$ (containing anticonformals ones) to a suitable Schottky uniformization of $S$ (that is, when $H$ is of Schottky type). If $H^{+}$is the index two subgroup of orientation preserving automorphisms of $H$ and $R=S / H^{+}$, then $H$ induces an anticonformal automorphism $\tau: R \rightarrow R$. If $\tau$ has fixed points, then we observe that $H$ is of Schottky type. If $\tau$ has no fixed points, then we provide a sufficient condition for $H$ to be of Schottky type. We also give partial answers for the excluded cases.
\end{abstract}

AMS Classification : 30F10, 30F40, 30F50

${ }^{*}$ Partially supported by projects Fondecyt 1030252, 1030373 and UTFSM 12.03.21. 


\section{Introduction}

Let us consider a closed Riemann surface $S$. An automorphism of $S$ is either a conformal or an anticonformal one. Let $H$ be a group of automorphisms of $S$. Retrosection theorem [2], [12] asserts that $S$ can be uniformized by a suitable Schottky group $G$, that is, a geometrically finite Kleinian group without parabolic transformations and isomorphic (as abstract group) to a free group of finite rank (equal to the genus of $S$ ). A Schottky uniformization of $S$ is a triple $(\Omega, G, P: \Omega \rightarrow S$ ), where $G$ is a Schottky group with region of discontinuity $\Omega$ and $P: \Omega \rightarrow S$ is a regular holomorphic covering with $G$ as covering group. The Schottky lifting problem is to decide the existence of some Schottky uniformization $(\Omega, G, P: \Omega \rightarrow S)$ of $S$ for which every automorphism $h \in H$ can be lifted, that is, there is an automorphism $\widehat{h}$ of $\Omega$ satisfying $P \widehat{h}=h P$. In case there is a Schottky uniformization for which $H$ lifts, we say that $H$ is of Schottky type. Since the region of discontinuity of a Schottky group is known to be a domain of class $O_{A D}$, such liftings are in fact restrictions of (extended) Möbius transformations [1].

In [10] we have provided a necessary and sufficient condition for a group $H$ of automorphisms to be of Schottky type. To describe that condition we need the following definition. A collection of pairwise disjoint simple loops on $S$, say $L_{1}, \ldots, L_{k} \subset S$, is called a Schottky system of loops of $H$ if

(1) each connected component of $S-\cup_{j=1}^{n} L_{j}$ is a genus zero bordered surface; and

(2) the collection of loops $\left\{L_{1}, \ldots, L_{k}\right\}$ is invariant under the action of the group $H$.

Theorem 1. A group $H$ of automorphisms of a closed Riemann surface $S$ of genus $g \geq 2$ is of Schottky type if and only if there is a Schottky system of loops of $H$.

A simple consequence of theorem 1 is the following reducibility necessary condition on a Schottky type group of automorphisms.

Corollary 1. If a group $H$ of automorphisms of a closed Riemann surface $S$ is not reducible, then it cannot be of Schottky type. 
To check the existence of such a collection of loops is in general not so easy to get. It is for that reason one would like to have some easy to verify conditions ensuring us the existence of a Schottky system of loops.

In [5] we have considered the case when $H$ only consists of conformal automorphisms and a simple to check necessary condition, called condition $(A)$, in order for $H$ to be of Schottky type was there obtained. In the same paper we prove that condition (A) is also a sufficient condition for the cyclic conformal case. Later, in a sequence of papers [6], [7] and [8], we have shown that condition (A) is also sufficient for Abelian groups, dihedral groups, the alternating groups $\mathcal{A}_{4}, \mathcal{A}_{5}$ and the symmetric group $\mathcal{S}_{4}$. In [8] was also observed that condition (A) is not a sufficient condition in general; for instance, the symmetric group $\mathcal{S}_{5}$ need some extra condition for it to be of Schottky type.

In [3] we had considered the case when $H$ is a cyclic group generated by some anticonformal automorphism $F: S \rightarrow S$, say of order $2 q$. On the quotient orbifold $R=S / H^{+}$we have an anticonformal involution $\tau: R \rightarrow$ $R$ induced by the automorphism $F$. If $\tau$ has fixed points (that is, $\tau$ is a reflection), then $H$ is of Schottky type [9]. If $\tau$ has no fixed points (an imaginary reflection), then we have obtained a condition, called condition (A-) (see below), which ensures $H$ to be of Schottky type. Later, in [10] we have provided a necessary and sufficient condition for $H$ to be of Schottky type in the case that $\tau$ is an imaginary reflection, completing the cyclic case. The following resume the cyclic anticonformal situation. The notion of a Klein-Schottky pairing can be found in [10] (as we do not need such a condition in this note, we do not recall the details).

Theorem 2 ([3, 10]). Let $S$ be a closed Riemann surface and $\psi: S \rightarrow S$ be an anticonformal automorphism of order $2 p$.

(i) If $p=2,3$, then $\psi$ is of Schottky type.

(ii) If $S / \psi$ has non-empty border, then $\psi$ is of Schottky type.

(iii) If no non-trivial power of $\psi$ has fixed points, then $\psi$ is of Schottky type.

Moreover, if $S / \psi$ has no boundary, then

(iv) $\psi$ is of Schottky type if and only if $\psi$ has a Klein-Schottky pairing.

(v) If $p$ is a prime, then $\psi$ is always of Schottky type. 
In this note we want to consider the case when $H$ is Abelian group and contains anticonformal automorphisms. As before, we denote by $H^{+}$its index two normal subgroup generated by the conformal automorphisms of $H$. We have an anticonformal involution $\tau: R \rightarrow R, R=S / H^{+}$, induced by any anticonformal automorphism in $H$. Clearly, we have that $\tau$ is a reflection if and only if there is a reflection $\eta \in H$. In this way, we are in a particular situation of [9] and we have that $H$ is of Schottky type. We reprove this for this particular case using a different topological argument which can be used in the case when the involution $\tau$ has no fixed points.

Theorem 3. Let $S$ be a closed Riemann surface and $H$ be an Abelian group of automorphisms of $S$ containing a reflection. Then $H$ is of Schottky type.

In the case that $H$ has no reflections, that is, $\tau$ is an imaginary reflection, we need to consider some extra condition, called condition (A-). First, we need to recall some generalities of a Schottky type group $H$.

Let $q>0$ be any odd integer number. An extended Möbius transformation $\eta$ which is conjugated to a transformation of the form

$$
\widehat{\eta}(z)=\frac{e^{k \pi i / q}}{\bar{z}}
$$

where $k \in\{1,3, \ldots, q-2\}$ is odd and relatively prime with $q$, is called an imaginary elliptic transformation. In this way, $\eta^{2}$ is an elliptic transformation of order $q$ and $\eta^{q}$ is an imaginary reflection. If $k=1$, then we say that $\eta$ is a geometric imaginary elliptic transformation. In any case, if $\eta$ is an imaginary reflection of order $2 q$, then $\widehat{\mathbf{C}} / \eta$ is a real projective plane with exactly one branch value of order $q$. When $q=1$, we are in the presence of imaginary reflections and $\widehat{\mathbf{C}} / \eta$ is a real projective plane without branch values.

Let us assume we have a Schottky type group $H$ of automorphisms of a Riemann surface $S$ of genus $g \geq 2$. Let $(\Omega, G, P: \Omega \rightarrow S)$ be a Schottky uniformization of $S$ for which $H$ lifts. We have the following facts.

1.- As the region of discontinuity $\Omega$ of a Schottky group is known to be a domain of type $O_{A D}$ [1], we have that for each $h \in H$, the lifted automorphism $\widehat{h}$ should be either the restriction of (i) an extended Möbius transformation if $h$ is anticonformal or (ii) a Möbius transformation if $h$ is conformal. In particular, the group $\widehat{G}$, generated by all the lifted transformations $\widehat{h}$, for all $h \in H$, contains $G$ as a finite 
index normal subgroup. If $\widehat{G}^{+}$denotes the subgroup of $\widehat{G}$ consisting of only the Möbius transformations, then we have, as $\widehat{G}^{+}$contains $G$ as a finite index normal subgroup, that $\widehat{G}^{+}$turns out to be a geometrically finite function group. Geometrically finite function groups have been classified by B. Maskit [14].

2.- As $\widehat{G}$ is a finite extension of $G$ and $G$ contains no parabolic transformations, then neither $\widehat{G}$ does.

3.- If $\widehat{h} \in \widehat{G}^{+}$is an elliptic transformation, then we know from [4] that either (i) both fixed points of $\widehat{h}$ belong to the region of discontinuity $\Omega$ of $G$ or (ii) there is a loxodromic transformation in $G$ commuting with $\widehat{h}$.

4.- If $\widehat{h} \in \widehat{G}^{+}$is an elliptic transformation, $\operatorname{Fix}(\widehat{h})=\{a, b\} \subset \Omega$ and there is some $\widehat{t} \in \widehat{G}$ so that $\widehat{t}(a)=b$, then the non-existence of parabolics in $\widehat{G}$ ensures that $\widehat{t}(b)=a$. It follows that: (i) if $\widehat{t} \in \widehat{G}^{+}$, then $\widehat{t}^{2}=I$; and (ii) if $\widehat{t} \notin \widehat{G}^{+}$, then $\widehat{t}$ is imaginary elliptic.

The above permits to construct a collection $\mathcal{F}$ formed of pairs $\{a, b\}$, so that:

(1) $a \neq b$;

(2) $a$ and $b$ are fixed points of the same elements in $H$;

(3) the rotation number (which are normalized to belong to $[-\pi, \pi)]$ ) of any conformal transformation in $H$, of order at least 3 , that fixes $a$ (then $b$ by (2)) is opposite to its rotation number at $b$;

(4) for each $a$ that is fixed by a non-trivial element of $H$, there is a $b$, also fixed by a non-trivial element of $H$, so that $\{a, b\} \in \mathcal{F}$;

(5) if $\{a, b\} \in \mathcal{F}$ are projected to the same point in $S / H^{+}$, then there is an element, say $j$, of order 2 in $H^{+}$that permutes $a$ with $b$. If $H$ is Abelian, then it follows that the stabilizer of $a$ in $H^{+}$is a cyclic group of order 2 commuting with $j$.

(6) if $\{a, b\} \in \mathcal{F}$ are projected to different points in $S / H^{+}$, but they projected to the same point in $S / H$, then there is an anticonformal element of $H$, say $\sigma$, permuting them. In this case, we have that $\sigma^{2}$ belongs to the stabilizer of $a$ in $H^{+}$. In the case that $H$ is Abelian, the property on the rotation numbers in (3) asserts that the stabilizer 
of $a$ in $\mathrm{H}^{+}$has order two and that the reflection either has order two or four.

If we restrict the above pairing so that there is no cases with situation (5) or (6), we get a simple condition as follows.

Condition (A-). Let $H$ be a group of group of automorphisms of a closed Riemann surface $S$ containing anticonformal automorphisms and containing no reflections. Let $R=S / H^{+}$and $\pi: S \rightarrow R$ be the holomorphic (branched) covering induced by $H^{+}$. We will say that $H$ satisfies condition $(A-)$ if all fixed points of the non-trivial elements of $H^{+}$can be arranged into pairwise disjoint pairs so that, if $\{a, b\}$ is one such pair, then:

(A1) $a \neq b, \pi(a) \neq \pi(b)$ and $\tau(\pi(a)) \neq \pi(b)$;

(A2) $H^{+}(a)=H^{+}(b)$, where $H^{+}(p)=\left\{f \in H^{+}: f(p)=p\right\}$; and

(A3) if $\left|H^{+}(a)\right|>2$ and $f$ is generator of $H^{+}(a)$, then the rotation number of $f$ at $a$ is opposite to the rotation number of $f$ at $b$ (we are using the normalization that the rotation number belongs to $(-\pi, \pi])$.

Remark 1. We must remark that this condition $\left(A_{-}\right)$is for groups containing anticonformal automorphisms and condition (A) (of the previous works) is for groups containing only conformal automorphisms.

Theorem 4. Let $S$ be a closed Riemann surface and $H$ be an Abelian group of automorphisms of $S$ containing no reflections. If $H$ satisfies condition (A-), then it is of Schottky type.

We may have that $H$ does not satisfies condition (A-) and neither contains reflections. We proceed to divide the fixed points of the non-trivial elements of $\mathrm{H}^{+}$into two disjoint sets, say $\mathrm{Set}_{1}$ and $\mathrm{Set}_{2}$, in order that the points in $S_{e} t_{1}$ can be paired to satisfy condition (A-) and the points in Set $_{2}$ can not be put into pairs satisfying the above condition. In other words, $S e t_{1}$ is maximal set of paired fixed points satisfying condition (A-). Of course, $S_{\text {et }}=\emptyset$ is already considered in the above result. We now assume Set $_{2} \neq \emptyset$. Observe that necessarily \#Set $t_{1}$ and $\#$ Set $_{2}$ are necessarily even as they are invariant under $H$ and we are assuming that on $H$ there are no reflections. 
Theorem 5. Let $S$ be a closed Riemann surface and $H$ an Abelian group of automorphisms, containing anticonformal but no reflections. If the genus of $S / H^{+}$is even and \#Set $2=2$, then $H$ is of Schottky type.

An example of non-Schottky type We proceed to show with one example that theorem 6 may be false if $\#$ Set $_{2}>2$. This example appears in [3], but the reason done in there to see that it is not of Schottky type is not quite clear. We proceed next to give another argument to obtain this. In [3] we have a closed Riemann surface $S$ of genus 8 together an anticonformal automorphism $F: S \rightarrow S$, of order 30, so that $S / F$ is the real projective plane with exactly two branch values of orders 3 and 5 , respectively. Let us assume we have a Schottky group $G$, with region of discontinuity $\Omega$ and a regular holomorphic covering $P: \Omega \rightarrow S$, with covering group $G$, for which $F$ lifts. Set $\widetilde{G}$ be the group generated by $G$ and any lift of $F$. Then $\widetilde{G}$ contains $G$ as a normal subgroup of index 30 and, in particular, it contains no pseudo-parabolic transformations. We denote by $\widetilde{G}^{+}$the index two subgroup of $\widetilde{G}$ consisting of its orientation preserving transformations. We have that $\widetilde{G}^{+}$then $G$ has no parabolic transformations and that $G$ is a normal subgroup of index 15 in $\widetilde{G}^{+}$. The quotient orbifold $\Omega / \widetilde{G}^{+}=S / F^{2}$ is the Riemann sphere with 4 branch values, two of them of order 3 and the other two with order 5 . In particular, $\widetilde{G}^{+}$has elliptic transformations of order 3 or 5 with fixed points in $\Omega$. Let $x \in \Omega$ be the fixed point of some elliptic transformation $E \in \widetilde{G}^{+}$. Denote by $y$ the other fixed point of $E$. As consequences of [11] and the fact that $\widetilde{G}^{+}$has no parabolic transformations, we have that $x, y \in \Omega$. Since $\Omega / \widetilde{G}$ has no two branch values of same order, we must have that $x$ and $y$ are equivalent under $\widetilde{G}$. In particular, there is some $T \in \widetilde{G}-\widetilde{G}^{+}$satisfying $T(x)=y$. We must also have that $T(y)=x$. In fact, if $T(y) \neq x$, then we will have two elliptic transformations $E$ and $T E T^{-1}$ in $\widetilde{G}^{+}$with exactly one common fixed point, a contradiction to discreteness of $\widetilde{G}^{+}$. It follows then that $T^{2} \in \widetilde{G}^{+}$fixes $x$ and $y$. All the above asserts that $T^{2}$ cannot be neither a loxodromic or parabolic transformation. The only possibility for $T^{2}$ is to be an elliptic transformation. It follows from discreteness of the group $\widetilde{G}$ that $T^{2}$ is elliptic of finite order and, in particular, $T$ is a pseudo-elliptic of finite order (anticonformal Möbius transformation of finite order). We have from the above that $E T=T E$ and that $\langle E, T\rangle$ uniformizes a real projective plane with exactly one branch value. In fact, if we normalize to have $x=0$ and $y=\infty$, then $T(z)=w / \bar{z}$, where $w \neq 1$ is some root of unity. We may also assume $E$ to be a geometric rotation, that is, $E(z)=e^{2 \pi i / n} z$, where $n \in\{3,5\}$. 
Now, set $U=T E \in \widetilde{G}-\widetilde{G}^{+}$. Since (i) $U^{2} \in \widetilde{G}^{+}$, (ii) $\widetilde{G}^{+}$only contains elliptic transformations of order 3 and 5 , and (iii) $U^{2}(z)=w^{2} e^{4 \pi i / n} z \in \widetilde{G}^{+}$, we must have $w^{2 n}=1$. Since $\Omega / \widetilde{G}$ is closed, we have $\langle E, T\rangle=\langle E, z \mapsto$ $-1 / \bar{z}\rangle$ and, in particular, we may assume $T(z)=-1 / \bar{z}$. In this way, for each elliptic transformation $E \in \widetilde{G}^{+}$there is an imaginary reflection $T \in \widetilde{G}-\widetilde{G}^{+}$ such that $T E=E T$ and that $\langle E, T\rangle$ uniformizes a real projective plane with exactly one branch value (either of order 3 or 5 ). It follows that we cannot produce a real projective plane with exactly two branch values with $\widetilde{G}$ and, in particular, $F$ cannot be a of Schottky type.

Theorem 6. Let $S$ be a closed Riemann surface and $H$ an Abelian group of automorphisms, containing anticonformal but no reflections. Assume that the genus of $R=S / H^{+}$is odd and $\# S_{\text {Set }}=2$. If we are able to choose simple closed curves $\gamma_{1}$ and $\gamma_{2}$ on $R$, each one invariant under the involution $\tau: R \rightarrow R$ so that one of them lifts to exactly $\left|H^{+}\right|$simple loops, then $H$ is of Schottky type.

Remark 2. For instance, if $H$ is a cyclic group of order 6 , generated by some anticonformal automorphism $F: S \rightarrow S$ so that $F^{3}$ is imaginary reflection, then the existence of the simple loops of the above theorem holds. But, there are cyclic groups of order 10 for which there are no simple loops satisfying the above.

Assume that we are in the above case and we are not able to get $\gamma_{1}$ and $\gamma_{2}$ in order that at least one of them can be lifted to $S$ into exactly $\left|H^{+}\right|$simple loops. In that case, we may require that another simple loop does it. Since we may only consider the case when genus of $R$ is one (after cutting along suitable dividing loops), we only need to set this case.

Theorem 7. Let $S$ be a closed Riemann surface and $H$ an Abelian group of automorphisms, containing anticonformal but no reflections. Assume that the genus of $R=S / H^{+}$is 1 and \#Set $2=4$. If we are able to choose nondividing simple closed curves $\gamma_{1}$ and $\gamma_{2}$ on $R$, each one invariant under the involution $\tau: R \rightarrow R$, and a simple closed curve $\delta$, disjoint from the above ones which separates the two branch values contained in the component of $R-\gamma_{1} \cup \gamma_{2}$ where is $\delta$ contained, and so that it lifts to exactly $\left|H^{+}\right|$simple loops, then $H$ is of Schottky type. 
Remark 3. Arguments similar to the example constructed above can be suitable modified in order to have a closed Riemann surface $S$ with an anticonformal automorphism $F: S \rightarrow S$ so that $S / F$ is the connected sum of two real projective planes and exactly 3 different branch values, say of orders 3, 5 and 7, and not of Schottky type. In particular, for \#Set $2 \geq 6$ theorem 7 in general fails.

\section{Proof of Theorems 3 and 4}

We first explain the main idea for finding the desired Schottky uniformization in each case. Assume we are given a group $H$ of automorphisms of some closed Riemann surface $S$, which may or not be Abelian. As before we set $R=S / H^{+}, \pi: S \rightarrow R$ the holomorphic (branched) covering induced by $H^{+}$and $\tau: R \rightarrow R$ the anticonformal involution induced by $H$ on $R$. As a consequence of theorem 1, we need to find a collection of Schottky loops for the Abelian group $H$ satisfying the respective hypothesis of theorems 3 or 4 . We need to take care of the branch values of $\pi: S \rightarrow R$.

Lemma 1. Let us assume we are in the hypothesis of either theorem 3 or theorem 4. Then we may find a pairwise disjoint collection $\mathcal{F}$ of simple loops on $R$, each loop $\gamma \in \mathcal{F}$ bounding a closed disc $\Delta_{\gamma} \subset R$ containing exactly two branch points of $\pi$, so that:

(i) $\mathcal{F}$ is invariant under the action of $\tau$;

(ii) each simple loop lifts to $\left|H^{+}\right|$simple loops on $S$; and

(ii) if $\Delta$ is one of the closed discs bounded by one of the loops, then $\pi^{-1}(\Delta)$ consists on genus zero bordered surfaces.

Proof. For the case that $\tau$ is an imaginary reflection, this exactly what condition (A-) asserts. When $\tau$ is a reflection, we have a simple closed loop $\beta$ formed by fixed points of $\tau$. If $p \in R$ is a branch value of $\pi$, then $q=\tau(p)$ is also. Take a simple $\operatorname{arc} \delta$ connecting them and so that it is invariant under $\tau$. A simple loop $\gamma$ surrounding $\delta$ will serve for our purposes.

We will use the following fact (consequences of [13]) during the rest of this section. 
Lemma 2. Let $S$ and $R$ closed Riemann surfaces and $\pi: S \rightarrow R$ be a holomorphic (branched) covering of degree $d$. Let $\gamma$ be a simple loop on $R$, passing through no branch value of $\pi: S \rightarrow R$ so that $\pi^{-1}(\gamma)$ consists of exactly $d$ simple loops. Let $R_{1}$ (respectively, $S_{1}$ ) be a component of $R-\gamma$ (respectively, $S-\pi^{-1}(\gamma)$ ) so that $\pi\left(S_{1}\right)=R_{1}$. Then we may find closed Riemann surfaces $\overline{R_{1}}, \overline{S_{1}}$ and conformal embeddings $I: S_{1} \hookrightarrow \overline{S_{1}}$, $J: R_{1} \hookrightarrow \overline{R_{1}}$, so that $\overline{R_{1}}-J\left(R_{1}\right)$ and $\overline{S_{1}}-I\left(S_{1}\right)$ are pairwise disjoint union of round discs, and the holomorphic (branched) covering $Q=\left.\pi\right|_{S_{1}}: S_{1} \rightarrow$ $R_{1}$ extends to a holomorophic (branched) covering $\bar{Q}: \overline{S_{1}} \rightarrow \overline{R_{1}}$ so that the branch values of $\bar{Q}$ are the same as for $Q$.

Proof. The embedding property is just consequence of the results in [13]. Since the complement of the embeddings are round discs, we may extend $Q$ to $\bar{Q}$ by using Schwarz's reflection principle. If $\delta$ is one of the circles on $\overline{S_{1}}$ bounding one of the round discs, that is, $\bar{Q}(\delta)=\gamma$, the hypothesis that $\pi^{-1}(\gamma)$ consists of exactly $d$ components, we have that $\bar{Q}$ is homeomorphism from $\delta$ to $\gamma$, then asserting that there are no branch values inside each of the rounded discs.

\subsection{Reduction to the free fixed points situation}

The lifting to $S$ of the collection of simple loops obtained from lemma 4 will give us a collection of pairwise disjoint simple loops, invariant under $H$. By cutting $S$ along these lifted loops, we obtain a collection of bordered surfaces

$S_{1}, \ldots, S_{r}$, permuted by the group $H$. Some of these surfaces are genus zero surfaces and we do not need to take care of them. Choose any of these, say $S_{1}$, which is not of genus zero. Consider $H_{1}=\left\{h \in H: h\left(S_{1}\right)=S_{1}\right\}$ and set $R_{1}=\pi\left(S_{1}\right)$. We need to find a collection of pairwise disjoint simple loops on $S_{1}$, invariant under $H_{1}$, so that they cut $S_{1}$ into genus zero surfaces. Since each loop of $\mathcal{F}$ lifts to exactly $\left|H^{+}\right|$simple loops, we may use lemma 5 to assume that the action of $H_{1}^{+}$is free fixed points. In this way, we get our first reduction: to assume the group $\mathrm{H}^{+}$acts free fixed points.

We proceed now to work each of the two cases, that is, depending if $\tau$ has or not fixed points, by separate.

\subsection{Case $\tau: R \rightarrow R$ is a Reflection}

In this case, our hypothesis is that $H$ contains some reflection $\eta: S \rightarrow S$ and $\pi \eta=\tau \pi$. Let us assume the genus of $R$ is equal to one, that is, $R$ is 
a torus. Then $S$ is also a torus and the lifting loops of one circle of fixed points of $\tau$ will give us a collection of invariant loops that divides $S$ into genus zero surfaces as desired.

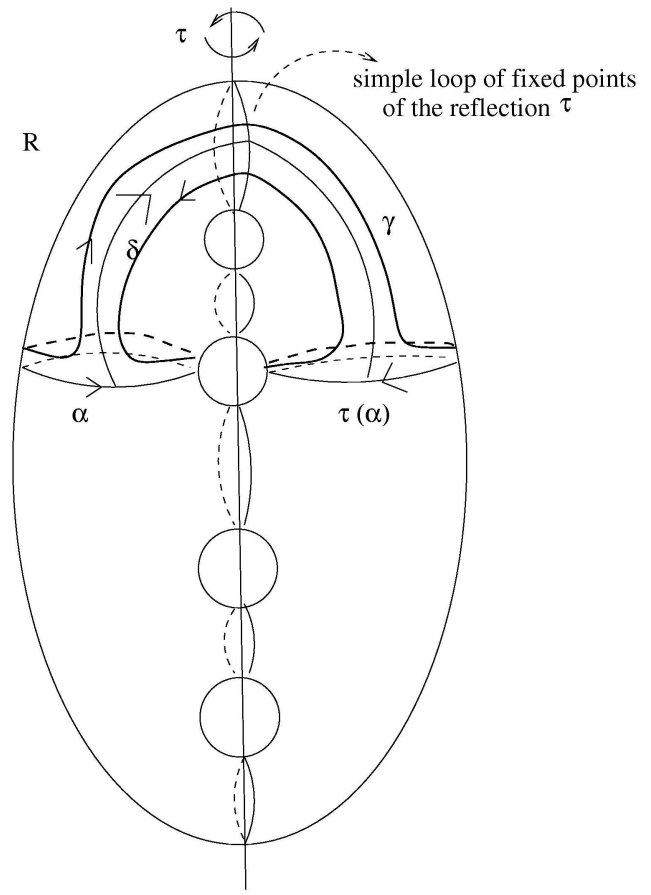

FIGURE 1

Let us assume that the genus of $R$ is at least two. We may consider a simple oriented loop $\alpha$ on $R$ so that $\tau(\alpha)$ is disjoint from $\alpha$ (see figure 1). Take a simple arc $\delta$ connecting these two loops which is $\tau$-invariant. We orient $\delta$ in order that it starts at $\alpha$ and ends at $\tau(\alpha)$. If $\alpha$ lifts to $\left|H^{+}\right|$simple loops (then also $\tau(\alpha)$ ), the lifting of $\{\alpha, \tau(\alpha)\}$ will give us an invariant set of pairwise disjoint simple loops, invariant under $H$. By cutting $R$ and $S$ along them, we get down in the genus of $R$. If $\alpha$ does not lift to exactly $\left|H^{+}\right|$simple loops, then the abelian property of $H$ asserts that by choosing any point $\pi(p) \in \alpha$, the lifts of $\alpha$ starting at $p$ ends at $T(p)$, some $T \in H^{+}$. 
Then the lifting of $\tau(\alpha)$ at $\eta(p)$ ends at $\eta(T(p))=T(\eta(p))$. We may assume that $\pi(p)$ is the starting point of $\delta$ (then $\tau(\pi(p))$ is the ending point of $\delta$ ). There is a simple loop $\gamma$ homotopic to $\alpha \cdot \delta \cdot \tau(\alpha)^{-1} \cdot \delta^{-1}$ which is $\tau$-invariant (see figure 1). The lifting of $\gamma$ at $p$ ends at $p$, that is, $\gamma$ lifts to exactly $\left|H^{+}\right|$ pairwise disjoint simple loops, invariant under $H$. We proceed to cut $R$ by $\gamma$ to go down in the genus of $R$ and to use lemma 5 to obtain theorem 3 by the above inductive steps.

\subsection{Case $\tau: R \rightarrow R$ is an imaginary Reflection}

If the genus of $R$ is one, then $S$ is again of genus one. In this case we may take any simple loop on $R$ which is $\tau$-invariant and then its lifting will be a pairwise disjoint collection of simple loops, invariant under $H$, dividing $S$ into genus zero surfaces. Let us assume the genus of $R$ is greater than one. We must take care of two subcases.

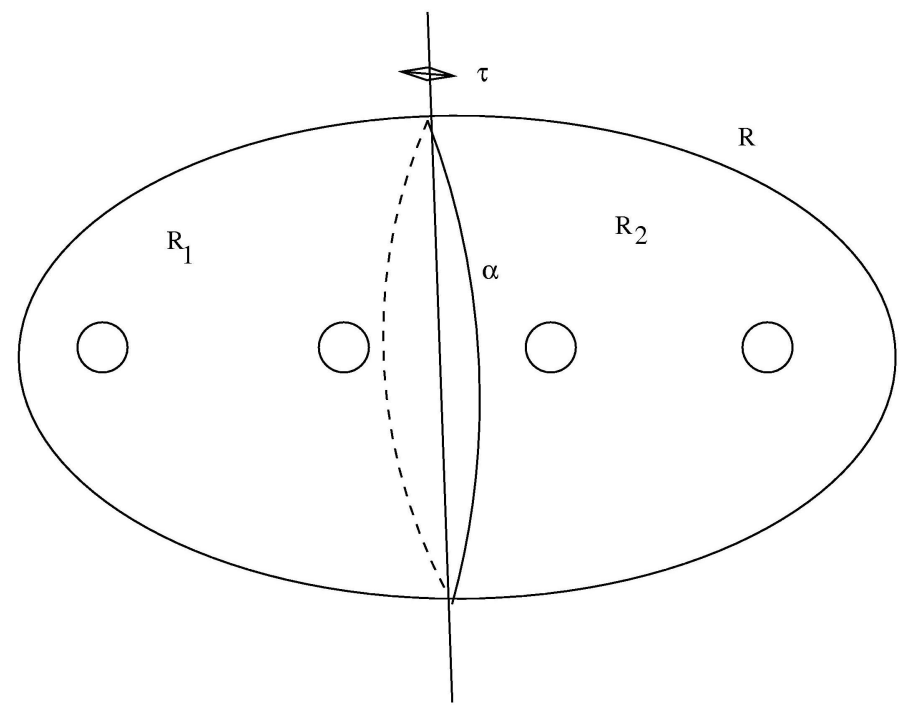

FIGURE 2

\subsubsection{Genus of $R$ is even}

In this case, there is a dividing simple loop $\alpha$ on $R$ which is $\tau$-invariant (see figure 2). This loop is a commutator and, since $H^{+}$is Abelian, it will lift to exactly $\left|H^{+}\right|$pairwise disjoint simple loops on $S$, invariant under $H$. In this way, by cutting $R$ along $\alpha$ we obtain two surfaces $R_{1}$ and $R_{2}$ 
of lower genus so that $\tau\left(R_{1}\right)=R_{2}$. Take a component $S_{1}$ of $\pi^{-1}\left(R_{1}\right)$. Since $\alpha$ lifts to exactly $\left|H^{+}\right|$simple loops, we may use lemma 5 to obtain closed surfaces $\overline{S_{1}}, \overline{R_{1}}$ and to extend the holomorphic covering as a regular Abelian covering. Denote by $H_{1}^{+}$the stabilizer in $H^{+}$of $S_{1}$. The arguments of [6] permits us to find a collection of pairwise disjoint simple loops on $S_{1}$, invariant under $H_{1}^{+}$, cutting it into genus zero surfaces. Translate these loops under $H$ to obtain a collection invariant under $H$ as desired.

\subsubsection{Genus of $R$ is odd}

In this case, there are two disjoint non-dividing simple loops $\alpha_{1}$ and $\alpha_{2}$ on $R$, each one $\tau$-invariant and both together dividing $R$ (see figure 3 ). Let $R_{1}$ and $R_{2}$ be We may draw a simple closed loop $\alpha$ on $R_{1}$ free homotopic to $\alpha_{1} \cdot \alpha_{2}$ (with the suitable orientations). We also consider the loops $\tau(\alpha)$ on $R_{2}$. The loop $\alpha$ divides $R_{1}$ into two surfaces, one of them is $R_{1,1}$ (which does not contains the borders $\alpha_{j}$ ). Since $\alpha$ is a commutator, it lifts to exactly $\left|H^{+}\right|$simple loops. We may proceed as in the above subcase for this surface (then also taking care of $\tau\left(R_{1,1}\right)$ ). The other surface is a threeholed sphere bounded by the loops $\alpha, \alpha_{1}$ and $\alpha_{2}$. Since $\alpha$ lifts to $\left|H^{+}\right|$ simple loops, then it is clear that the lifting of this surface are genus zero surfaces.

\section{The Excluded Cases: theorems 5, 6 and 7}

The excluded cases are given when $H$ satisfies the following two properties:

(1) $H$ does not contains reflections and

(2) $H$ does not satisfies condition (A-).

On the quotient surface $R$ it is possible to draw a simple closed curve $\alpha$ so that $\tau(\alpha)$ is disjoint from it and both together divide $R$ into three surfaces $R_{1}, R_{2}$ and $\tau\left(R_{1}\right)$, where:

(i) $R_{1}$ is a closed Riemann surface with one boundary, say $\alpha$, and containing no branch values of $\pi$;

(ii) $R_{2}$ is invariant under $\tau$, has exactly two boundaries, $\alpha$ and $\tau(\alpha)$, contains all the branch values of $\pi$ and it has

(ii.1) genus zero if the genus of $R$ is even;

(ii.2) genus one if the genus of $R$ is odd. 


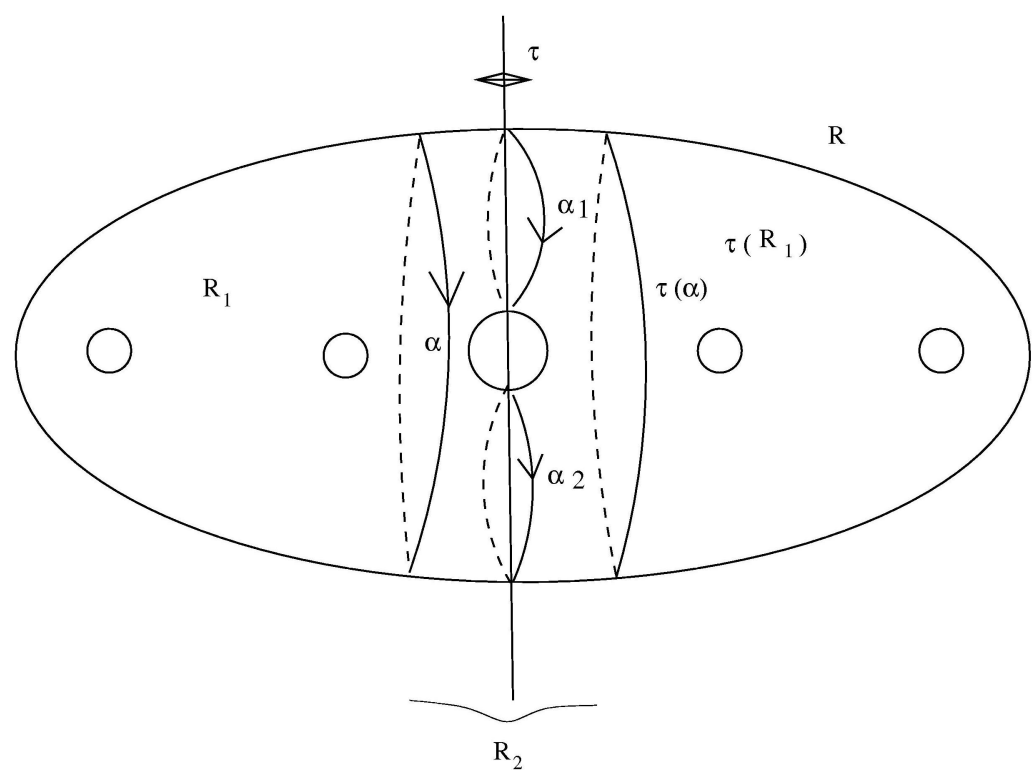

FIGURE 3

\section{First reduction: Can assume $R$ has genus 0 or 1 .}

For $R_{1}$ we may proceed as in [6] to find a collection of pairwise disjoint simple loops on $\pi^{-1}\left(R_{1}\right)$, invariant under $H^{+}$, and dividing $\pi^{-1}\left(R_{1}\right)$ into genus zero surfaces. On $\pi^{-1}\left(\tau\left(R_{1}\right)\right)$ we use the $H$-translates of the loops in $\pi^{-1}\left(R_{1}\right)$. As a consequence, by lemma 3 , we only need to study the case when $R$ is either of genus 0 or 1 .

\section{Permuting Branch values in $R$.}

Assume first that $R$ has genus 0, we have may choose a simple loop $\gamma$ which is invariant under $\tau$. If $p_{1}, p_{2}$ are branched values of $\pi: S \rightarrow R$ that belong to the same component disc of $R-\gamma$, then $\tau\left(p_{1}\right), \tau\left(p_{2}\right)$ belong to the other component disc. We may change the simple loop $\gamma$ by a new one, say $\gamma^{\prime}$, which is also $\tau$-invariant and so that $p_{1}$ and $\tau\left(p_{2}\right)$ belong to the same component disc of $R-\gamma^{\prime}$. Similarly, for $R$ of genus 1, we have may choose two disjoint simple loop $\gamma_{1}, \gamma_{2}$, each one invariant under $\tau$. If $p_{1}, p_{2}$ are branched values of $\pi: S \rightarrow R$ that belong to the same component annulus of $R-\left(\gamma_{1} \cup \gamma_{2}\right)$, then $\tau\left(p_{1}\right), \tau\left(p_{2}\right)$ belong to the other component annulus. We may change the simple loop $\gamma_{1}$ by a new one, say $\gamma_{1}^{\prime}$, which 
is also $\tau$-invariant and disjoint from $\gamma_{2}$, so that $p_{1}$ and $\tau\left(p_{2}\right)$ belong to the same component annulus of $R-\left(\gamma_{1}^{\prime} \cup \gamma_{2}\right)$.

\section{Reducing Branch values in $R$.}

Assume first that $R$ has genus 0 and we have chosen a simple loop $\gamma$ invariant under $\tau$. If we have two branch values, say $p, q$ in the same component disc $\Delta$ of $R-\gamma$, a simple loop $\alpha$ defining a disc inside $\Delta$ containing $p$ and $q$ and no other branch value so that the loop $\alpha$ lifts to exactly $\left|H^{+}\right|$loops, then the components of $\pi^{-1}(\Delta)$ are genus zero surfaces. Similarly, assume $R$ has genus 1 and we have chosen two simple loops $\gamma_{1}$ and $\gamma_{2}$, each one invariant under $\tau$. If we have two branch values, say $p, q$ in the same component annulus $\Delta$ of $R-\left(\gamma_{1} \cup \gamma_{2}\right)$, a simple loop $\alpha$ defining a disc inside $\Delta$ containing $p$ and $q$ and no other branch value so that the loop $\alpha$ lifts to exactly $\left|H^{+}\right|$loops, then the components of $\pi^{-1}(\Delta)$ are genus zero surfaces.

It follows from the above that we may assume also that once we have chosen $\gamma$ for $R$ of genus 0 (or $\gamma_{1}, \gamma_{2}$ for $R$ of genus 1 ), and $\Delta$ is one of the two components of $R$ after deleting these loops, then:

(3) there is no a simple loop surrounding exactly two branch points in $\Delta$ which lifts to exactly $\left|H^{+}\right|$loops.

Of Course, the above condition (2) asserts that on $R$ we must have branch values. Let $2 k(k \geq 1)$ be the number of branch values in $R$, say $p_{1}, \ldots, p_{k}$ are in one of the two components (then, $\tau\left(p_{1}\right), \ldots, \tau\left(p_{k}\right)$ are in the other component). Now we proceed to describe some partial answer in this exclude cases.

\subsection{Genus of $R$ is even}

Case $k=1$ : Proof of theorem 5 If $R$ has genus zero and $k=1$, then Riemann-Hurwitz's formula asserts that $H^{+}$is a cyclic group and $S$ has genus zero and we have in this case proved theorem 5 .

\subsection{Genus of $R$ is odd}

Case $k=1$ : Proof of Theorem 6 The existence of the loops $\gamma_{1}$ and $\gamma_{2}$ on $R$, as in the hypothesis of theorem 6 , permits us to use similar arguments as in the previous case. 
Case $k \geq 2$ : Proof of theorem 7 For $k=2$ and $S / F$ being the connected sum of two real projective planes and exactly two branch values we get the result.

Remark 4. We think that a necessary and sufficient condition similar to the one given for cyclic groups generated by anticonformal automorphisms as in [10] may be given for the Abelian groups containing no reflections.

\section{References}

[1] L. V. Ahlfors and L. Sario, Riemann Surfaces Princeton Univ. Press. Princeton, New Jersey, 1960.

[2] L. Bers, Automorphic forms for Schottky groups, Adv. in Math. 16, pp. 332-361, 1975.

[3] R.A. Hidalgo and A.F. Costa. Anticonformal automorphisms and Schottky coverings. Ann. Acad. Scie. Fenn. 26, pp. 489-508, 2001.

[4] R.A. Hidalgo. The mixed elliptically fixed point property for Kleinian groups. Ann. Acad. Sci. Fenn. Series A.I. Math. 19, pp. 247-258, 1994.

[5] R.A. Hidalgo. On Schottky groups with automorphisms. Ann. Acad. Scie. Fenn. Ser. AI Mathematica 19, pp. 259-289, 1994.

[6] R.A. Hidalgo. Schottky uniformizations of closed Riemann surfaces with abelian groups of conformal automorphisms. Glasgow Math. J. 36, pp. 17-32, 1994.

[7] R.A. Hidalgo. Dihedral groups are of Schottky type. Revista Proyecciones 18, pp. 23-48, 1999.

[8] R.A. Hidalgo. $\mathcal{A}_{4}, \mathcal{A}_{5}, \mathcal{S}_{4}$ and $\mathcal{S}_{5}$ of Schottky type. Revista Matematica Complutense 15, 2002.

[9] R.A. Hidalgo. Real surfaces, Riemann matrices and algebraic curves. Contemporary Mathematics 311, pp. 277-299, 2001.

[10] R.A. Hidalgo. Automorphisms groups of Schottky type. preprint. 
[11] R.A. Hidalgo. The mixed elliptically fixed point property for Kleinian groups. Ann. Acad. Scie. Fenn. Ser. AI Mathematica 19, pp. 247-258, 1994.

[12] P. Koebe. Über die Uniformisierung der Algebraischen Kurven II, Math. Ann. 69, pp. 1-81, 1910.

[13] B. Maskit. Canonical domains on Riemann surfaces. Proc. of the Amer. Math. Soc. 106, pp. 713-721, 1989.

[14] Maskit, B. On the classification of Kleinian Groups II-Signatures. Acta Mathematica 138, pp. 17-42, 1977.

\section{Ruben A. Hidalgo}

Departamento de Matemática

Universidad Técnica Federico Santa María

Casilla 110 - V

Valparaíso

Chile

e-mail : ruben.hidalgo@mat.utfsm.cl 